\title{
Disambiguation of Overlapping Experiences by Neurons in the Medial Entorhinal Cortex
}

\author{
Paul A. Lipton, ${ }^{1,3}$ John A. White,,$^{2,3}$ and Howard Eichenbaum ${ }^{1,3}$ \\ Departments of ${ }^{1}$ Psychology and ${ }^{2}$ Biomedical Engineering, and ${ }^{3}$ Center for Memory and Brain, Boston University, Boston, Massachusetts 02215
}

\begin{abstract}
Hippocampal neuronal activity distinguishes separate events that share common elements. Here, we examined whether the capacity to disambiguate overlapping experiences is an exclusive feature of hippocampal processing or whether information processing one stage earlier in the hippocampal system also disambiguates common elements of distinct experiences. We compared the spatial firing patterns of neurons in the dorsocaudal medial entorhinal cortex (dcMEC) and hippocampal CA1 neurons in animals continuously alternating left-turn and right-turn routes through a T-maze. Neurons in the dcMEC more strongly distinguished left-turn from right-turn trials compared with CA1 neurons, whereas CA1 neurons more selectivity encoded places traversed within each route. These results indicate that dcMEC spatial firing patterns are experience dependent and reflect the mnemonic demands of a spatial memory task. Furthermore, the results suggest that neuronal populations in the dcMEC and CA1 differentially emphasize complementary aspects of spatial memory representations.
\end{abstract}

Key words: medial entorhinal cortex; hippocampus; spatial navigation; memory; T-maze; episodic memory

\section{Introduction}

Episodic memory depends on the capacity to form and retrieve distinct representations of experiences that share overlapping elements (Tulving, 1983). Convergent evidence from human neuroimaging (Kumaran and Maguire, 2006), animal models (Agster et al., 2002; Gilbert and Kesner, 2006), single neuron recordings (Frank et al., 2000; Wood et al., 2000; Ferbinteanu and Shapiro, 2003; Lee et al., 2006), analyses of immediate early gene expression patterns (Vazdarjanova and Guzowski, 2004), and computational models (Levy, 1996; Lisman, 1999) identify the medial temporal lobe region, and in particular, the hippocampus, as critical to the disambiguation of memories for overlapping experiences.

Characterization of neural activity during spatial memory performance has provided insights into how hippocampal circuits distinguish experiences that share spatial and behavioral events (Eichenbaum et al., 1999). Thus, several studies have shown that CA1 neurons fire differentially as rats traverse common locations within overlapping routes (see Fig. 1) (Frank et al., 2000; Wood et al., 2000; Ferbinteanu and Shapiro, 2003). Comparing spatial coding properties throughout the hippocampal system in animals performing spatial memory tasks will yield additional insights about how the capacity for disambiguation emerges within hippocampal neural circuits.

Recent findings on the dorsocaudal medial entorhinal cortex

Received Nov. 13, 2006; revised April 23, 2007; accepted April 24, 2007.

This work was supported by National Institute of Mental Health Grants MH51570 and MH071702 and Office of Naval Research Grant N00014-05-1-0392. We thank M. Hasselmo, J. Chrobak, and J. Manns for comments on a previous version of this manuscript and J. Manns and A. McCaffrey for technical assistance.

Correspondence should be addressed to Dr. Howard Eichenbaum, Department of Psychology, Center for Memory and Brain, 2 Cummington Street, Boston University, Boston, MA 02215. E-mail: hbe@bu.edu.

D0I:10.1523/JNEUROSCI.1063-07.2007

Copyright $\odot 2007$ Society for Neuroscience $\quad$ 0270-6474/07/275787-09\$15.00/0
(dcMEC) suggest an important role for this area in spatial information processing within the hippocampal system. The dcMEC receives a greater proportion of neocortical projections carrying visuospatial, posterior parietal, and temporal association and direct CA1 inputs than other regions of the medial or lateral entorhinal cortex (Burwell, 2000; Kerr et al., 2007), and preferentially sends direct projections to the dorsal hippocampus (Witter et al., 1989; Dolorfo and Amaral, 1998). Lesions to the dcMEC, but not the ventromedial MEC, disrupt retention and retard new learning of a water-maze task (Steffenach et al., 2005). In rats foraging for food pellets distributed randomly throughout an open environment, dcMEC neurons exhibit spatial coding resembling hippocampal place cells, representing position, head direction, and velocity (Fyhn et al., 2004; Hafting et al., 2005; Hargreaves et al., 2005; Sargolini et al., 2006). Earlier studies also reported that medial entorhinal neurons exhibit location-specific activity (Mizumori et al., 1992; Quirk et al., 1992), including the identification of neurons that disambiguate overlapping routes (Frank et al., 2000). Importantly, however, these neurons were located in anterior and ventral regions of the MEC in contrast to the recently characterized dorsocaudal band noted for its specialized spatial processing (Hafting et al., 2005).

As noted above, dcMEC firing properties were derived exclusively as animals explored open fields, leaving unknown their response properties in animals performing a spatial memory task. Here, we recorded as rats performed a continuous spatial alternation task that allows us to determine whether dcMEC activity, like that of hippocampal neurons, reflects task demands for disambiguation of overlapping experiences, or whether dcMEC neurons exclusively represent spatial information. We found that neurons in both areas exhibit location-related firing, as well as differentiation of left- and right-turn trials. Furthermore, dcMEC neurons more robustly disambiguate left and right trial types, 


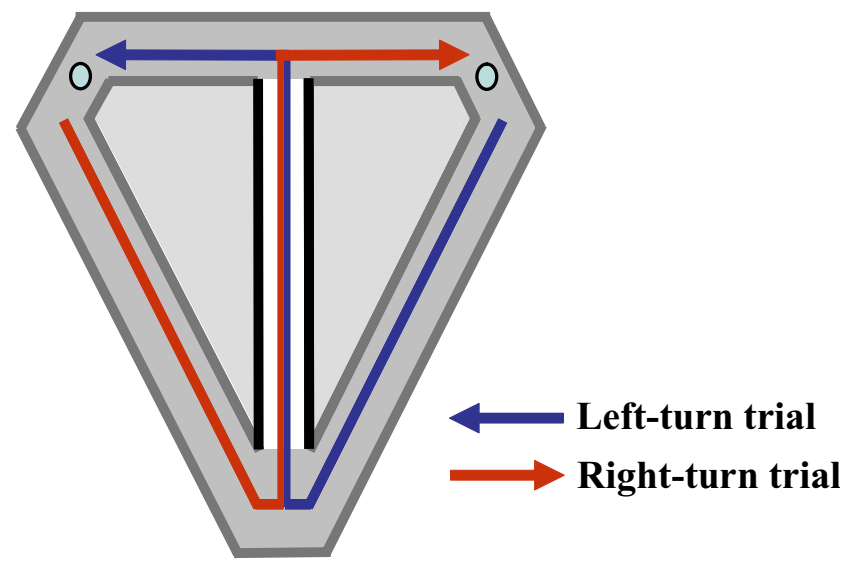

Figure 1. Schematic of modified T-maze. Small circles at upper left and right corners represent reward sites.

whereas hippocampal neurons more selectively encode locationrelated information.

\section{Materials and Methods}

Subjects. Subjects were eight male Long-Evans rats weighing between 425 and $450 \mathrm{~g}$. All surgical and euthanasia procedures followed National Institutes of Health guidelines and approved Institutional Animal Care and Use Committee protocols. Each of five rats were implanted with six tetrodes aimed directly at the dorsal CA1 region of the hippocampus, and each of three rats were implanted with 13 tetrodes aimed at the dorsocaudal region of the MEC. Food was available ad libitum to all animals throughout the entire study. Water access was restricted to $30 \mathrm{~min}$ each day before training and recording sessions. Rats were housed individually and kept on a $12 \mathrm{~h}$ light/dark cycle. All training and testing occurred during the light phase of the cycle, $\sim 5 \mathrm{~d}$ /week.

Apparatus. A modified T-maze, constructed entirely of wood and painted with a nonreflective black paint, consisted of three distinct areas: a central stem, choice arms, and return arms (Fig. 1). The central stem of the maze was $130 \mathrm{~cm}$ long and $5.7 \mathrm{~cm}$ wide. The distal end of the central stem led directly into the adjoining left and right choice arms, each 38.5 $\mathrm{cm}$ long and $10.0 \mathrm{~cm}$ wide. At the end of each choice arm was a $1.5-\mathrm{cm}-$ diameter circular well (depth of $0.4 \mathrm{~cm}$ ) where water rewards were delivered. Return arms connected the goal areas back to the initial portion of the central stem and were $128.0 \mathrm{~cm}$ long and $10.0 \mathrm{~cm}$ wide. Along the entire maze were $4.3-\mathrm{cm}$-high walls. The maze was elevated $76.2 \mathrm{~cm}$ and enclosed on three sides by floor to ceiling length curtains onto which distinctive cues were fastened, whereas the fourth side that lay adjacent to the start area was open to a wall with additional cues. The cues and their positions remained constant throughout the study.

Behavioral testing. Animals were shaped to perform the continuous alternation in successive stages before electrode implantation as described previously (Wood et al., 2000). In the first stage, each rat was placed in the initial start area of the central stem. The rat was allowed to run in only one direction along the central stem toward the choice point. At the choice point, a black Plexiglas barrier blocked entry to one goal arm forcing the rat to make either a left or a right turn and then advance to the well and receive a reward. Next, the rat ran along the return arm back to the start area to initiate another trial. An additional Plexiglas barrier blocked entry into the opposite return arm, forcing the rat to run along the central stem once again toward the choice point. Once at the choice point, the black Plexiglas barrier was now positioned to force the rat to select the choice arm blocked on the previous trial. Positioning of the barriers in this manner was repeated throughout the first stage of training to direct the rat toward alternating choice arms and eliminate the possibility of making errors or retracing steps. The animal was directed in this manner for 30-40 trials/d for $>3-4 \mathrm{~d}$. In the second stage of training, barriers to the choice arms were eliminated, providing the rat with the option of either choice arm. The rat received a water reward only if it correctly alternated between left and right choice arms; only during this stage was the animal allowed to self-correct by retracing his steps to the choice point and choosing the alternate arm without having to return to the start area. If the animal chose to proceed down the return arm without self-correcting, he was thereafter rewarded for alternating. This stage lasted until the animal successfully alternated on $\geq 80 \%$ of the trials, which required $1-5 \mathrm{~d}$. In the third stage of training, all remaining barriers were eliminated. Animals rarely attempted to turn around and run in the opposite direction or choose the opposite return arm rather than run down the central stem. Once trained, the animals were allowed to perform 40-70 continuous trials in each session. During testing, incorrect choices were never rewarded, and if committed, the rat was required to continue through the maze and select the alternate arm to receive a water reward.

Electrophysiology: electrodes. Tetrodes were composed of four twisted $12.5 \mu \mathrm{m}$ insulated nichrome wires (California Fine Wire, Grover Beach, CA). Each tetrode was individually drivable and part of a custom-made head stage assembly. Multiple electrodes were chronically implanted and directed at either the dorsal CA1 $(3.2 \mathrm{~mm}$ posterior and $1.9 \mathrm{~mm}$ lateral to bregma) or dcMEC ( $8.8 \mathrm{~mm}$ posterior and $4.8 \mathrm{~mm}$ lateral to bregma, angled $12^{\circ}$ anteriorly in the sagittal plane). Electrophysiological data were acquired using a 64-channel system built around custom amplifiers (Boston University Electronics Design Facility) and the real-time Linux program, Spike (written by Loren Frank, University of California at San Francisco, San Francisco, CA). Extracellular spikes were differentially amplified by a gain of 10,000 , digitized at $31.25 \mathrm{kHz}$, and filtered at $600-6000 \mathrm{~Hz}$ (300-6000 Hz for MEC units). The lower cutoff for MEC recordings prevented unnecessary reduction of some wide MEC waveforms (Frank et al., 2001). A single tetrode within the hippocampus or entorhinal cortex served as a reference. This system also acquired synchronized digital video images that tracked the location of three incandescent bulbs arrayed over the head stage and assigned $x$ and $y$ coordinates to a centroid calculated from the three bulbs.

After recovery, animals were placed in a 16-inch-diameter circular holding chamber with a 12-inch-high wall and screened for unit activity. At the end of each recording session, or if no units were identified, tetrodes were advanced in $\sim 80-100 \mu \mathrm{m}$ intervals. When recordings were completed in each animal, a $40 \mu \mathrm{A}$ current was passed through each tetrode for $10-25$ s to create variably sized lesions to mark the final position of the electrode tips. The animal was then perfused with a potassium ferrocyanide solution to facilitate localization of electrode tips and tracks. Recording locations were estimated by measuring backwards from the final electrode position along the electrode track.

Data analysis. Unit isolation was achieved off-line using Off-line Sorter (Plexon, Dallas, TX). Only cells with a mean peak firing rate $>1 \mathrm{~Hz}$ on the central stem of the T-maze were included in the analysis. Data from any unit recorded over multiple recording sessions were analyzed only once and taken from the first session in which they were isolated. Only clusters with clearly defined boundaries were characterized as individual units and included in subsequent analyses (for dcMEC example clusters and waveforms, see supplemental Fig. 1, available at www.jneurosci.org as supplemental material). Additionally, cluster boundaries and waveform parameters for all units included in the analysis remained stable over the entire recording session.

Spatial firing patterns. The pattern of spatial firing throughout the entire recording session was characterized using Place Field Analysis software (Matthew Shapiro, Mount Sinai School of Medicine, New York, $\mathrm{NY}$ ). The program divides the maze into $2.0 \times 2.0 \mathrm{~cm}$ pixels and calculates the firing rate within each pixel as the total number of spikes divided by the total time spent in that pixel across the entire session. Firing rate was calculated only for periods when the rat was moving $\geq 2 \mathrm{~cm} / \mathrm{s}$. A place field was characterized as a minimum of eight contiguous pixels, touching either at the corner or edge, in which the firing rate exceeded $20 \%$ of the peak rate, estimated as the highest firing rate observed in any pixel on the maze (Muller et al., 1987).

A set of descriptive statistics were used to characterize and compare spatial firing patterns within and across brain regions. Spatial information rate, measured in bits per second (Skaggs et al., 1993), describes how much information about spatial location each place field conveys. A 
A.

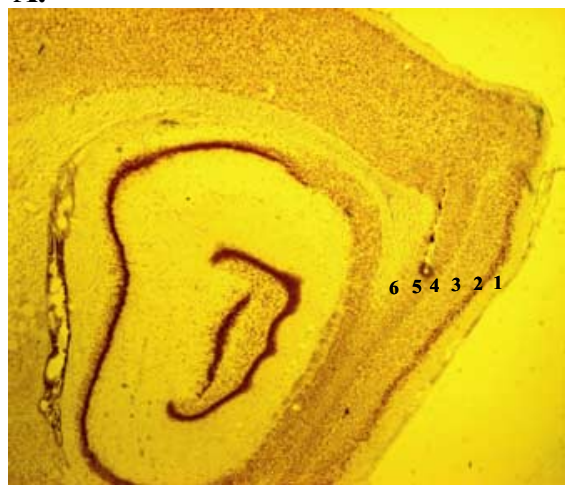

B.

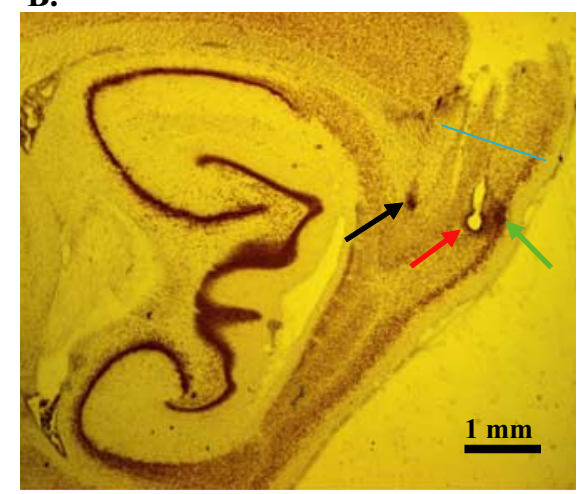

trials at each value of $\mathrm{x}$, and assumed that $p[r \mid \mathrm{L}, \mathrm{x}]$ was normally distributed with this mean and variance. The function $p[r \mid \mathrm{R}, \mathrm{x}]$ was calculated the same way from right-turn trials.

For each cell, log-likelihood ratios, $\ln \{p[r \mid \mathrm{L}, \mathrm{x}] / p[r \mid \mathrm{R}, \mathrm{x}]\}$, were summed over all central stem bins for each trial. In cases in which the log-likelihood sum is greater than zero, maximum likelihood analysis predicts that the data came from a left-turn trial; otherwise, a right-turn trial is predicted. Here, we report the average absolute value of the summed log-likelihood ratio; larger values of this term indicate firing-rate patterns that are statistically more distinct.

In addition, we calculated and report three additional values that further describe differences in firing properties among CA1 and MEC neurons. The first measure, $p_{\text {correct }}$, is based on a maximum-likelihood guess performed for each trial compared against the actual outcome of that trial. To calculate $p_{\text {correct }}$, we performed

higher rate implies greater information content. Spatial tuning, measured as the ratio of infield to outfield firing rate, parallels spatial information rate such that a higher tuning ratio implies greater specificity for individual spatial locations. Place field size is a measure of the number of contiguous $2 \mathrm{~cm}^{2}$ pixels with a firing rate within an individual place field that exceeds $20 \%$ of the overall peak rate (Muller et al., 1987). Smaller place fields arguably convey more information about individual spatial locations within an environment and thus are a complement to higher spatial information rates and spatial tuning ratios. Each measure was calculated for every place field that occurred on the maze.

Disambiguation of left- and right-turn trials. To identify those cells whose activity reflected the disambiguation of left- and right-turn trial episodes, each cell that fired along the central stem was subjected to an additional set of analyses. To start, the activity of each cell that fired when the rat traversed the stem was divided into correct left- and right-turn trials; incorrect trials were excluded from the analysis. Individual units were isolated before the separation of trials, therefore both trial types contained units defined by identical waveform parameters and cluster boundaries.

The central stem was subdivided into seven $12.7 \mathrm{~cm}$ segments. Unit activity was considered only within those segments in which the paths during left- and right-turn trials overlapped. Path overlap was determined by a paired $t$ test of average lateral position within each segment. For this analysis, rather than calculate firing rate on a pixel by pixel basis, rate was calculated as the number of spikes that occurred as the rat passed through each $12.7-\mathrm{cm}$-long, 5.7-cm-wide segment divided by the amount of time within that segment.

A two-way ANOVA compared the firing rates of each cell between leftand right-turn trial types and segments as independent (fixed) variables (Wood et al., 2000). A significant main effect of trial type or a trial type by segment interaction identified a cell as differentiating left-turn from right-turn trials. However, a significant main effect of segment in the absence of a significant main effect of trial type or trial type by segment interaction reflected location-specific activity only.

The results of a two-way ANOVA only reveal that neural activity during left-turn and right-turn trials differs, rather than measuring the degree to which they differ. Therefore, to quantitatively compare the incidence and robustness of trial disambiguation, we calculated a loglikelihood ratio (Dayan and Abbott, 2001), a value that indicates the difference in firing patterns between left-turn and right-turn trials, for every neuron. A larger number reflects firing patterns that are more easily distinguishable across the trial type. The log-likelihood ratio was calculated as follows: $\ln \{p[r \mid \mathrm{L}, \mathrm{x}] / p[r \mid \mathrm{R}, \mathrm{x}]\}$, where $p[r \mid \mathrm{L}, \mathrm{x}]$ is the probability density function of left-turn (L) trials at position $\mathrm{x}$, evaluated at the observed firing rate $r$, and $p[r \mid \mathrm{R}, \mathrm{x}]$ is the equivalent function for rightturn (R) trials (Dayan and Abbott, 2001). To find $p[r \mid \mathrm{L}, \mathrm{x}]$, we divided data sets from left-turn trials into bins and estimated the rate of each cell on each trial in each bin. We calculated the mean and variance over all a maximum likelihood analysis using the conditional density functions as described above. $p_{\text {correct }}$ represents the number of times that prediction was correct, divided by the total number of trials. Therefore, $p_{\text {correct }}$ is the average trial-by-trial probability that the log-likelihood analysis gives the correct answer for each trial type.

The second measure, $p_{\text {chance, }}$ is the average probability that firing patterns across left-turn and right-turn trials arose by chance, given a $p_{\text {correct }}$ of 0.5 (i.e., the firing rate contains no trial-specific information). To calculate $p_{\text {chance }}$, we evaluated the following formula: Pnk $=n ! / k ! /(n-$ $k) !\left(0.5^{k}\right) 0.5^{(1-k)}$, where $n$ is the number of trials, and $k$ is the number of apparently correct answers from maximal likelihood analysis. To get $p_{\text {chance }}$, we summed Pnk for all values of $k$ greater than or equal to the number associated with our measured value of $p_{\text {correct. }}$. A $p_{\text {chance }}<0.05$ determined that a cell could successfully distinguish trial type. As a third measure, we calculated the square root of the average variance in firing rate for each cell type, which yields a measure of trial by trial variability of firing rate within a given trial type.

\section{Results}

Three rats with electrodes implanted in the dorsocaudal region of the MEC performed an average of 47.9 trials in nine recording sessions with a mean accuracy of $85.2 \%$ correct turns. Five rats with electrodes implanted in the CA1 performed an average of 53.4 trials in 10 recording sessions with a mean accuracy of $88.5 \%$. The total number of trials completed and performance accuracy did not differ among dcMEC and CA1 animals (trials: $F_{(1,18)}=0.76, p=0.18$; accuracy: $\left.F_{(1,18)}=1.12, p=0.3\right)$.

Of 209 neurons recorded in dcMEC, 154 had at least one place field on the maze. Postmortem histological analysis verified that 120 units were isolated from superficial layers II and III and 34 from deep layers V and VI, all within a range of $\sim 50-1000 \mu \mathrm{m}$ from the postrhinal border (Fig. 2 and supplemental Fig. $2 \mathrm{~A}$, available at www.jneurosci.org as supplemental material). Fortyone cells had place fields along the central stem (33 of these cells were in layer II and III; eight in layers V and VI). Of 146 CA1 neurons recorded, 124 had at least one place field and 48 of these were active as the rat traversed the central stem. Three dcMEC and two CA1 units with place fields on the central stem were excluded from any further analysis because of significant path deviation (paired $t$ test; $p<0.05$ ) across left-turn and right-turn trials through central stem segments within which their place fields fell. 


\section{Firing patterns of dcMEC and CA1 neurons}

Consistent with recent characterizations by Hargreaves et al. (2005) and Fyhn et al. (2004), dcMEC neuronal activity was associated with the animal's location in the environment. Here we extend the observation of spatial firing in dcMEC neurons to rats performing a spatial memory task and report that the activity of neurons in the dcMEC also reflect mnemonic demands beyond the perceptual experience of the maze environment (Quirk et al., 1992). Furthermore, the prevalence and strength of trial disambiguation and place is complementary in dcMEC and CA1, such that dcMEC cells disambiguate trial type more strongly than CA1 neurons, whereas CA1 neurons encode discrete places more selectively than dcMEC neurons.

We will first compare the firing patterns of several example dcMEC and CA1 neurons, then present quantitative analyses on the full set of neurons recorded in both areas. Figures 3 and 4 present examples of dcMEC and CA1 neuronal activity, respectively, as the rats traversed the central stem of the maze. Results of the twoway ANOVA and log-likelihood analyses for each of these units is detailed in Table 1. These analyses simultaneously characterize the spatial selectivity of each cell and the extent to which it differentiated leftturn and right-turn trials.

The firing patterns of four dcMEC neurons illustrated in Figure 3 reflect the range of spatial and trial-type specificity observed among the population of dcMEC neurons, and all of these example cells were active as the rat traversed the central stem of the maze. The example cell shown in Figure $3 A$ was selectively and robustly active on the lower portion of the central stem and fired equally strongly on left-turn and right-turn trials. The cell shown in Figure $3 B$ was active as the rat traversed the first half of the stem, earlier on right-turn trials and later on left-turn trials. The neuron shown in Figure $3 C$ was predominantly active as the rat traversed early portions of the stem on right-turn trials. Figure $3 D$ shows a cell that was active along the entire central stem, with a significantly higher firing rate on right-turn trials.

In contrast to dcMEC neurons, the activity of CA1 neurons was more highly localized, yielding more discrete place fields, and generally reflected weaker disambiguation of left-turn and rightturn trials. The examples shown in Figure 4 reflect the range of spatial and trial-type specificity observed in CA1 cells. The cell in Figure $4 A$ was active both in the middle and at the end of the stem, and the cell shown in Figure $4 B$ had a single place field in the middle of the central stem; neither of these units fired differentially during left-turn or right-turn trials. The cell shown in Figure $4 C$ had a localized firing field on the central part of the stem, and was more robustly active on right-turn trials than on left-turn trials. The cell shown in Figure $4 D$ had a single small place field on the top part of the stem and was preferentially active on right turn trials. Importantly, we did not observe any CA1 activity that resembled the poorly localized, trial-type specific dcMEC firing patterns of the type that extended throughout the central stem (Fig. 3C,D). We did, however, observe highly specific location-related firing in dcMEC that resembled CA1 firing patterns (Fig. $3 A, B$ ). In contrast to previous reports that describe differences in place field size and spatial information content that vary as a function of ventral distance from the postrhinal border (Fyhn et al., 2004; Hafting et al., 2005), we observed no such significant relationship for either measure $\left(R^{2}=0.003, p>0.7\right.$; $R^{2}=0.09, p>0.05$, respectively) (supplemental Fig. $2 B, C$, available at www.jneurosci.org as supplemental material). 
A.
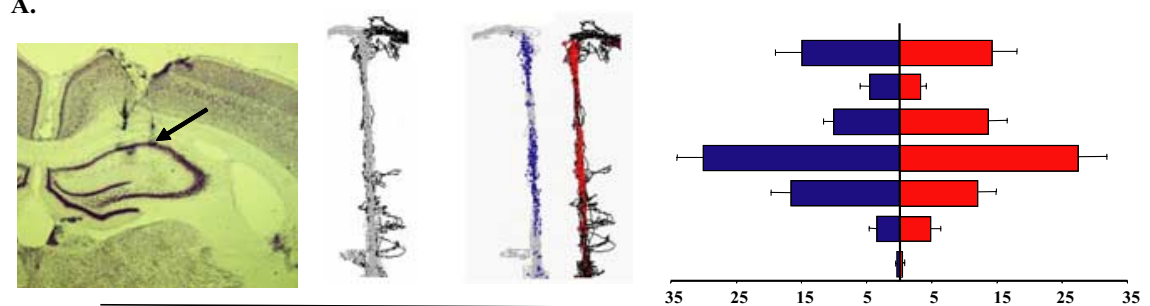

B.
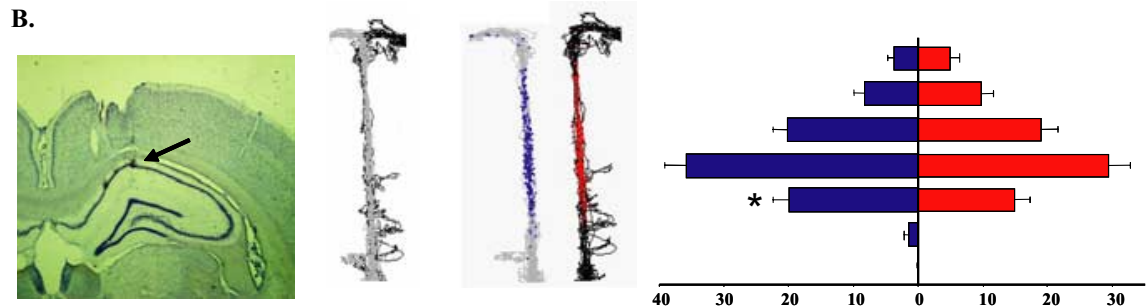

C.
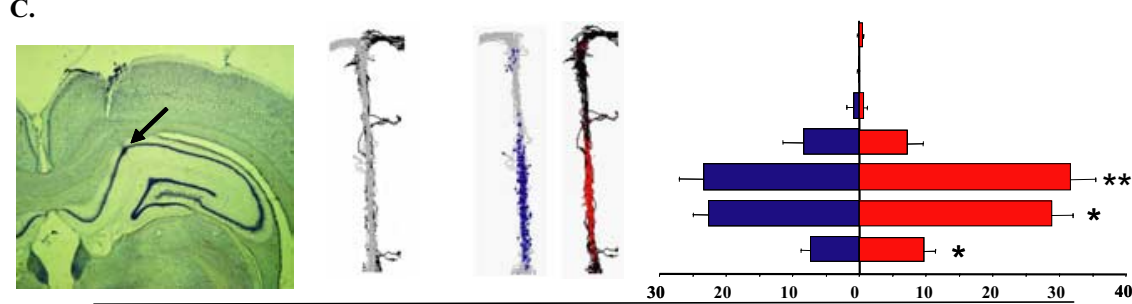

D.
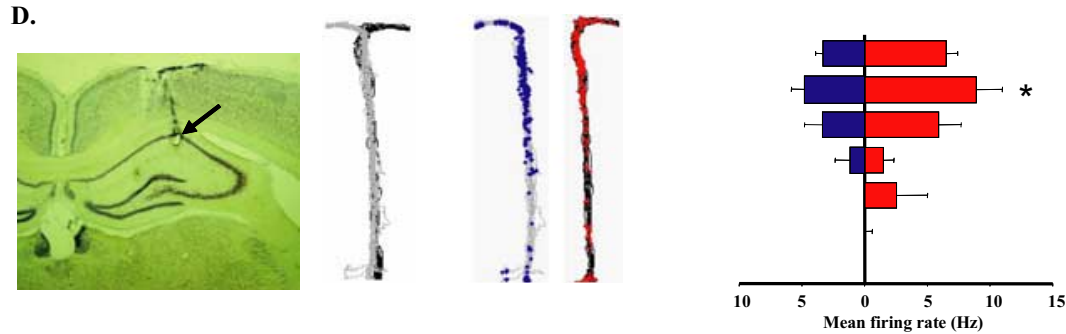

Figure 4. Hippocampal activity that represents a range of spatial and trial-type selective firing patterns. Refer to caption in Figure 3 for description of panels. Black arrows point to recording site for example units.

\section{Quantitative analysis of spatial selectivity in dcMEC and CA1 neurons}

The superior spatial selectivity of hippocampal neurons, apparent in the comparison of example cells, was confirmed in standard quantitative analyses of spatial selectivity, including place field size, spatial tuning ratio, and mean spatial information rate (for these measures on the dcMEC and CA1 example cells presented in Figs. 3 and 4, see Table 1). The average size of a dcMEC place field was $330.8 \mathrm{~cm}^{2}$, which was larger than that for CA1 cells (256.8 $\mathrm{cm}^{2}$; Wilcoxon rank-sum test, $p<0.0003$ ), and the total area of all place fields of dcMEC neurons was $832 \mathrm{~cm}^{2}$, which was larger than that for CA1 neurons $\left(590.9 \mathrm{~cm}^{2}\right.$; Wilcoxon ranksum test, $p<0.02$ ). A comparison of the distributions of place field size indicates that a substantial proportion of dcMEC neurons had very large areas of spatially related firing relative to the distribution of place field sizes in hippocampal neurons (Fig. 5A).

Furthermore, the degree of spatial tuning, measured as the ratio of firing rates inside versus outside a place field, was markedly higher for CA1 cells (11.5) than for dcMEC neurons (3.0; Wilcoxon rank-sum test, $p<8.8 \mathrm{E}-16$ ). A comparison of the distribution of spatial tuning ratios illustrates that CA1 neurons have a broad range of spatial tuning ratios, whereas a large proportion of dcMEC neurons have very low spatial tuning (Fig. 5B). In addition, with the exception of a significant difference in place field size between all superficially isolated dcMEC units and all deep dcMEC units (superficial, $313.1 \mathrm{~cm}^{2}$; deep, $395.5 \mathrm{~cm}^{2}$; Wilcoxon rank-sum test, $p<0.05$ ), comparisons between information rates (superficial, 0.91 bits/s; deep, $0.83 \mathrm{bits} / \mathrm{s}$ ) and tuning ratios (superficial, 3.1 ; deep, 2.7) revealed no significant differences between superficial (Wilcoxon rank-sum test, $p=0.737$ ) and deep (Wilcoxon rank-sum test, $p=0.9791$ ) dcMEC layers. This pattern is consistent with recent characterizations of neuronal activity recorded from the dorsocaudal band of the MEC (Sargolini et al., 2006).

The differences in spatial selectivity were also observed in the ANOVAs performed on each cell. Each of the 48 CA1 neurons that fired when the rat was on the maze stem was selectively active within specific segments of the stem (i.e., main effect of segment), whereas only 30 of 41 dcMEC neurons showed this spatial selectivity. Furthermore, of 16 CA1 cells that also significantly differentiated the two trial types, all had spatially selective firing on the stem, whereas of 23 dcMEC cells that differentiated trial types, only 12 fired selectively along the stem.

The impact of these differences in spatial selectivity for spatial coding can be quantified by the amount of information (in bits per second) conveyed by the firing rate of a neuron across localized regions of the maze (Skaggs et al., 1993). Mean spatial information rates for dcMEC neurons in rats performing the current spatial alternation task averaged $0.89 \mathrm{bits} / \mathrm{s}$, whereas for CA1 neurons, the average information rate was significantly higher at $2.02 \mathrm{bits} / \mathrm{s}$ (Wilcoxon rank-sum test, $p<0.00001$ ). A comparison of the distribution of spatial information rates indicates that a greater proportion of CA1 place fields convey more spatial information than corresponding dcMEC place fields (Wilcoxon ranksum test, $p<0.01$ ) (Fig. 5C). Because different studies calculate and characterize spatial firing using slightly different parameters, such as pixel size or spatial information rate in bits per spike (Skaggs et al., 1996; Hargreaves et al., 2005) rather than bits per second (Skaggs et al., 1993; Fyhn et al., 2004), a direct comparison across studies is often difficult. Consequently, a rudimentary comparison between the current study and Fyhn et al. (2004) reveals that spatial information rates for our dcMEC neurons closely match what Fyhn et al. (2004) (0.91 bits/s) report from their recordings of animals randomly foraging for food in large enclosures. Interestingly, however, CA1 neurons in the current task provided considerably higher spatial information than that observed for their CA1 neurons (1.1 bits/s).

\section{Quantitative analysis of trial disambiguation in dcMEC and \\ CA1 neurons}

The ANOVAs identified 23 of the 41 dcMEC neurons with place fields on the central stem (18 superficial; five deep) that fired differentially during left-turn and right-turn trials. By comparison, 16 of 48 CA1 neurons that fired when the rat was on the maze stem distinguished left-turn and right-turn episodes. To quanti- 
Table 1. Quantitative measures of spatial firing patterns, log-likelihood estimates, and two-way ANOVA results for each dcMEC and hippocampal unit presented in Figures 4 and 5 , respectively

\begin{tabular}{|c|c|c|c|c|c|c|c|c|c|}
\hline Unit & $\begin{array}{l}\text { Information } \\
\text { rate (bits/s) }\end{array}$ & $\begin{array}{l}\text { Spatial } \\
\text { tuning }\end{array}$ & $\begin{array}{l}\text { Place field } \\
\text { size }\left(\mathrm{cm}^{2}\right)\end{array}$ & $\begin{array}{l}\text { Log-likelihood } \\
\text { ratio }\end{array}$ & $P_{\text {correct }}$ & $P_{\text {chance }}$ & Main effect of trial type & Main effect of segment & Interaction \\
\hline $\operatorname{dcMEC}(\text { unit A) })^{a}$ & 1.9 & 5.6 & 244 & 2.5 & 0.72 & 0.006 & ns & $F_{(6,251)}=44.43 ; p<0.00001$ & ns \\
\hline $\operatorname{dcMEC}(\text { unit B) })^{a}$ & 1.3 & 5.5 & 389 & 0.94 & 0.64 & 0.02 & ns & $F_{(6,405)}^{(0,23)}=15.88 ; p<0.00001$ & $F_{(6,405)}=3.75 ; p<0.0012$ \\
\hline dcMEC (unit C) ${ }^{a}$ & 0.5 & 1.8 & 512 & 2.25 & 0.67 & 0.05 & $F_{(1,209)}=8.1 ; p<0.0049$ & $F_{(6,209)}=11.75 ; p<0.00001$ & $F_{(6,209)}^{(0,70)}=5.11 ; p<0.0001$ \\
\hline dcMEC (unit D) $)^{a}$ & 0.3 & 2.0 & 540 & 4.3 & 0.76 & 0.00005 & $F_{(1,405)}=13.2 ; p<0.05$ & $F_{(6,405)}=2.57 ; p<0.0055$ & ns \\
\hline CAl (unit A) & 1.8 & 9.0 & 300 & 0.6 & 0.58 & 0.2 & $\mathrm{~ns}$ & $F_{(6,265)}=68.5 ; p<0.00001$ & ns \\
\hline CAI (unit B) ${ }^{a}$ & 2.6 & 7.6 & 248 & 0.65 & 0.68 & 0.02 & ns & $F_{(6,265)}^{(0,20)}=25.69 ; p<0.00001$ & ns \\
\hline $\operatorname{CAl~(unit~C)~}^{a}$ & 3.7 & 11.8 & 220 & 1.2 & 0.58 & 0.13 & $F_{(1,461)}=6.61 ; p<0.01$ & $F_{(6,461)}^{(0,26)}=104.8 ; p<0.00001$ & $F_{(6,461)}=2.52 ; p<0.03$ \\
\hline $\mathrm{CAI}_{\text {(unit D) }}{ }^{a}$ & 1.4 & 4.0 & 280 & 2.7 & 0.58 & 0.12 & $F_{(1,419)}=27.79 ; p<0.00001$ & $F_{(6,419)}=34.39 ; p<0.00001$ & $F_{(6,419)}=3.5 ; p<0.002$ \\
\hline
\end{tabular}

Each dcMEC unit distinguished left-turn and right-turn trials: A by log-likelihood; B, C, and D by 2-way ANOVA and log-likelihood estimation. Hippocampal units B, C, and D distinguished left-turn and right-turn trials: B by log-likelihood; $C$ and D by two-way ANOVA.

${ }^{a}$ Unit that distinguished left-turn and right-turn trials.

A. Place Field Size Distribution [Wilcoxon rank-sum test : $p=0.0003$ ]

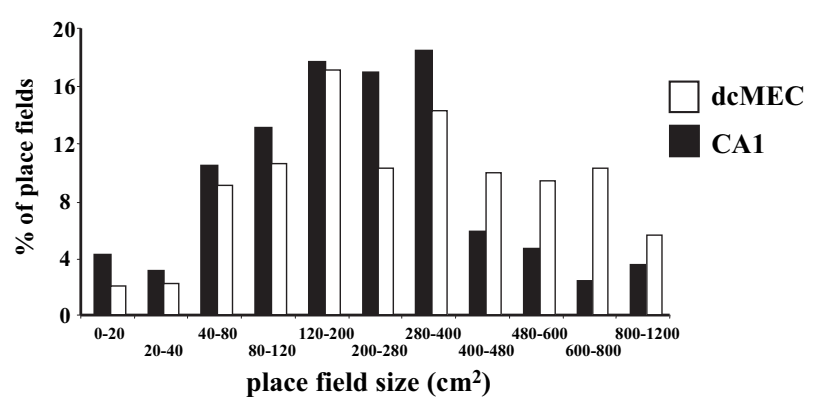

B. Spatial Tuning Ratio Distribution [Wilcoxon rank-sum test: $p<7.7 \mathrm{E}-16$ ]

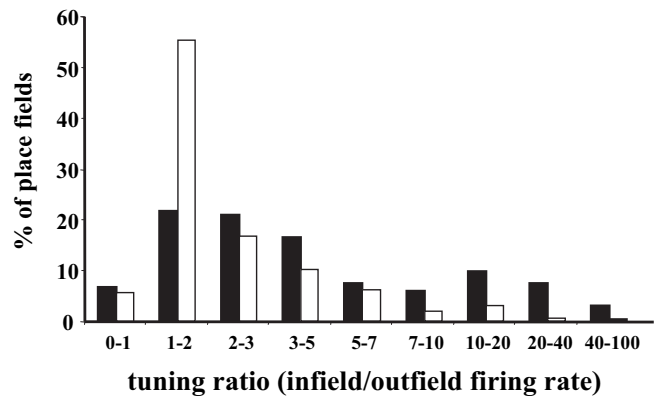

C. Spatial Information Rate Distribution [Wilcoxon rank-sum test: $p=0$ ]

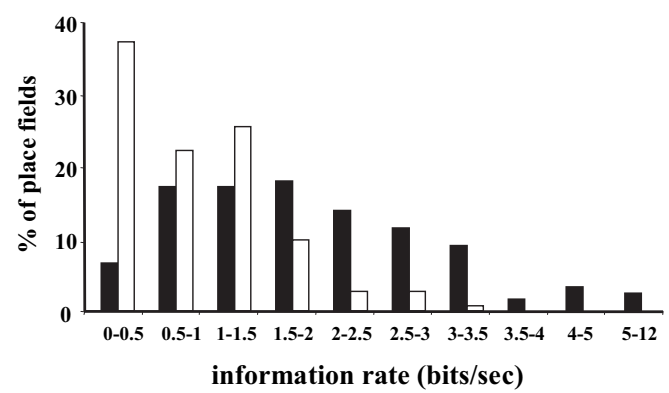

Figure 5. Distribution of measures of spatial selectivity for all dcMEC and CA1 units with place fields. $A$, Place field size. $B$, Spatial tuning ratio. $C$, Spatial information content.

tatively measure and compare the incidence and robustness of trial disambiguation among dcMEC and CA1 neurons, we used a set of analyses based on log-likelihood estimation (see Materials and Methods). The average log-likelihood ratio is a statistic that reflects the dissimilarity of firing patterns of a cell as the rat passes through the central stem on left-turn and right-turn trials. On average, log-likelihood ratios for dcMEC neurons with place

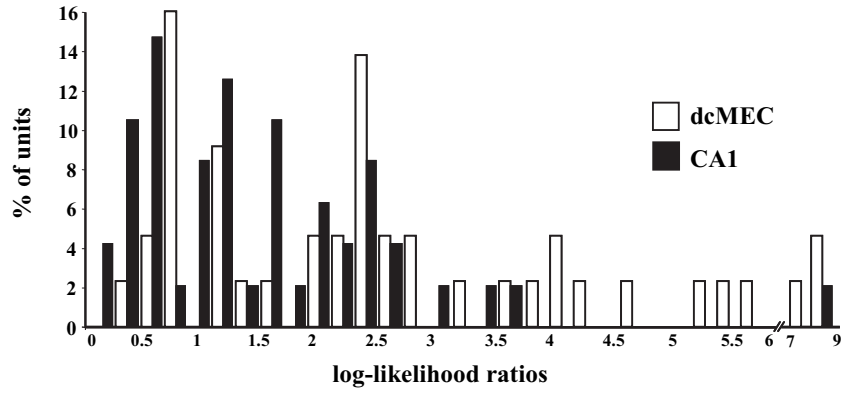

Figure 6. Distribution of log-likelihood ratios for all dCMEC and CA1 units with place fields on the central stem. The different patterns reflect that a higher proportion of dcMEC units more clearly distinguish between left- and right-turn trials and are thus better predictors of trial type. Wilcoxon rank-sum test, $p<0.003$.

fields on the central stem were significantly greater than for CA1 neurons (dcMEC, 2.82; CA1, 1.7; Wilcoxon rank-sum test, $p<$ 0.003). Accordingly, comparing the distributions of loglikelihood ratios reveals that more dcMEC neurons than CA1 neurons had higher scores, and many dcMEC neurons had the highest scores observed (Fig. 6). Log-likelihood ratios were also used to calculate the probability of predicting trial type correctly $\left(p_{\text {correct }}\right)$ on each trial based on the similarity of its firing pattern to the set of patterns generated on left- and right-turn trials. The average $p_{\text {correct }}$ for dcMEC neurons was $70 \%$, whereas CA1 neurons were significantly less successful, correctly predicting trial type on only $63 \%$ of the trials (Wilcoxon rank-sum test, $p<$ $0.0001)$. Also, to determine whether the log-likelihood scores reflect statistically different firing patterns between left- and right-turn trials, we calculated the average probability ( $\left.p_{\text {chance }}\right)$ that the difference in activity patterns between left- and rightturn trials occurred by chance; as noted above, a $p_{\text {chance }}$ of less than or equal to 0.05 was considered significant. By this measure, the proportion of dcMEC neurons that successfully distinguished left- and right-turn trials was $90 \%$, whereas only $50 \%$ of CA1 neurons successfully distinguished trial type (Wilcoxon ranksum test, $p<0.0001$ ). Finally, we also measured the trial-to-trial reliability of firing rate for each trial type by calculating the square root of the summed variance in firing rate and averaging over leftand right-turn trials. The variability of firing in dcMEC neurons was lower on average $(4.15 \mathrm{~Hz})$ compared with that for CA1 neurons $(6.3 \mathrm{~Hz})$. Together, these analyses indicate that the firing patterns of individual dcMEC neurons more clearly differentiate left- and right-turn trial types, are better predictors of trial type, and are more reliably active from trial to trial within the set of leftor right-turn trials than CA1 neurons, supporting the conclusion 
that dcMEC representations more strongly distinguish between left- and right-turn routes.

The ANOVA and log-likelihood calculations did not always identify the same cells as distinguishing left- and right-turn trials. All of the 23 dcMEC neurons that were identified by ANOVA were also identified by log-likelihood estimation as distinguishing trial type. In addition, the log-likelihood estimation identified as differentiating trial type 14 of the 18 dcMEC neurons not recognized by ANOVA. In contrast, only seven of the 16 CA1 neurons identified as differentiating trial types by ANOVA were correspondingly identified by the log-likelihood measure, whereas 17 of the 32 CA1 neurons not identified by ANOVA were identified by the log-likelihood calculation as distinguishing trial types. The discrepancy between analysis methods may derive from a consideration of firing reliability, such that log-likelihood ratios are disproportionately affected by trials in which firing rates near zero are more commonly observed in hippocampal neurons, whereas dcMEC neurons are more reliably active from trial to trial as indicated by the smaller square root of the summed variance for dcMEC neurons as noted above. A combination of the ANOVA and log-likelihood estimations provides the most stringent criterion for trial-type differentiation. The two methods identified 23 of 41 dcMEC cells that fired on the maze stem and differentiated the two trial types. In contrast, only seven of 48 CA1 neurons that fired as the rat passed through the stem differentiated the two trial types.

\section{Discussion}

Consistent with previous reports (Frank et al. 2000; Wood et al., 2000), a substantial proportion of CA1 neurons distinguished between left- and right-turn trial episodes. Is trial disambiguation a function that occurs initially within the hippocampus? The present results strongly suggest that this may not be the case. Instead, we found that a substantial proportion of neurons in dcMEC, a cortical area that provides major afferents to the hippocampus, had place fields on the central stem that strongly distinguished left- and right-turn trials even as the rat traversed identical locations and engaged in the same behaviors. Indeed, the proportion of cells that distinguished trial types and the strength of this differentiation was greater in dcMEC than in CA1 neurons, a finding supported by two different statistical measures. At the same time, consistent with recent reports (Fyhn et al., 2004; Hargreaves et al., 2005), we observed that a substantial proportion of dcMEC neurons, like hippocampal neurons, encoded locations traversed by rats performing a spatial memory task. However, a larger proportion of CA1 neurons had locationspecific activity and exhibited greater spatial selectivity than dcMEC neurons. Thus, the present findings indicate that the disambiguation of overlapping experiences occurs before the hippocampus, and suggest distinctions in the processing functions of medial entorhinal and hippocampal neurons in the representation of overlapping routes.

In addition, the present results contrast with recent studies that argue that dcMEC spatial representations code exclusively for an experience-independent spatial coordinate system (Sargolini et al., 2006). For example, the grid structure reportedly remains stable after environmental manipulations, such as cue rotation, lighting, enclosure size (Hafting et al., 2005), or color of the enclosure walls (Fyhn et al., 2007). Just as hippocampal spatial correlates encode more than just spatial features (Wood et al., 2000; Ferbinteanu and Shapiro, 2003), their immediate inputs from dcMEC likewise exhibit experience-dependent spatial representations.
Before considering the theoretical implications of these results, we consider two additional aspects of the current findings that differ from those of previous reports. First, whereas Fyhn et al. (2004) report that neurons in dorsal CA1 and dcMEC display a similar degree of spatial specificity, in the present study, neurons in CA1 exhibited significantly greater spatial selectivity than in dcMEC. The superiority of hippocampal neurons in spatial coding was evident in multiple measures, including the overall proportion of cells with place fields, the size of place fields, spatial tuning ratios, and spatial information content. The difference between the current and previous results likely rests in distinctions between behavioral protocols used. In the earlier studies, rats were simply required to forage for randomly distributed food pellets. In contrast, accurate performance in the current behavioral task required animals to remember the preceding experience or the goal of the current episode. Thus, the current results suggest that the memory demands of the current spatial task may have sharpened hippocampal processing of discrete, spatially defined events. That hippocampal cells show less of a distinction between trial types suggests that hippocampal processing focuses more on individual locations that compose each route than on differences between the two trial types. Alternatively, hippocampal representations that are similar between the two trial types may serve to bridge the two kinds of experience, supporting the network of memories that compose the entire task (Eichenbaum et al., 1999). Notably, although the hippocampus is not essential to perform the continuous spatial alternation task (Ainge et al., 2007), hippocampal neuronal activity typically reflects spatial and other features of an array of behavioral tasks whether or not hippocampal function is required (Muller et al., 1987; Markus et al., 1995). In the present task, hippocampal and entorhinal activity clearly reflect both the spatial features of the environment and the task demands for disambiguation of distinct experiences.

Second, we observed a lower proportion (18-33\%, depending on analysis) of CA1 neurons that distinguished left- and rightturn trials than a previous study (67\%) (Wood et al., 2000) of animals performing the same continuous spatial alternation. Other studies using similar tasks report variable proportions of hippocampal neurons that disambiguate overlapping spatial experiences. For example, the proportion of hippocampal neurons that distinguished left- and right-turn trials in a spatial alternation task very similar to the current one was $70 \%$ (Lee et al., 2006). In that study, however, animals were initially trained to complete only left- or right-turn trials, rather than to alternate. This manner of training may establish a representation of the trial type on which the animal is initially trained, which then persists relatively unaltered despite the introduction of the second trial type, and therefore produces a greater percentage of neurons that disambiguate trial type. In contrast, the proportion of hippocampal neurons that differentiated routes in different spatial alternation tasks performed on a W-shaped track was 36\% (Frank et al., 2000), whereas the proportion that differentiated specific routes by origin and destination on a plus maze was 59\% (Ferbinteanu and Shapiro, 2003). Two additional studies likewise report variable percentages of differential hippocampal activity, one that used a spatial sequence task (37-68\%, depending on training day), and the other a Y-maze spatial alternation in which all arms served as both goal and start arms (zero) (Lenck-Santini et al., 2001; Bower et al., 2005). Thus, the proportion of hippocampal neurons that distinguish overlapping routes varies substantially across studies and may depend on as yet unclear distinctions in the task, the training protocol, or the statistical methods used to identify differential firing patterns. We cannot exclude the possi- 
bility that this variability may be a factor in the different percentages of dcMEC and CA1 neurons that disambiguate trial type. However, it neither explains nor detracts from our primary finding that varying mnemonic demands imposed over behavioral and environmental stability inspires both dcMEC and CA1 neurons to differentially encode trial type, and to do so in a complementary manner.

\section{A model of hippocampal information processing that underlies spatial memory performance}

Recent findings have indicated that neurons in multiple areas within the hippocampus (Leutgeb et al., 2007) as well as other brain areas (Freedman et al., 2003) can be differentiated by their performance in pattern separation. So, it may be more important to not ask whether different components of the hippocampal system perform pattern separation but how the differences in trial disambiguation and spatial representation by neurons observed here provide insights about the functional organization of this system.

Recent models provide a framework for understanding the present observations in the context of this spatial memory task (Hasselmo and Eichenbaum, 2005; Howard et al., 2005). To accurately perform the continuous spatial alternation, an animal must selectively retrieve information about the immediately preceding trial episode independent of many other similar previous left- or right-turn episodes. The temporally defined context of the most recent trial provides the most salient dimension along which an immediately preceding episode can be differentiated from other previous trials. In Hasselmo and Eichenbaum's (2005) model, the entorhinal cortex performs two functions that underlie the associative retrieval of a previous sequence that characterizes the route taken on the preceding trial. First, based on the observation of persistent spiking activity in layer II of the entorhinal cortex in slice preparations (Klink and Alonso, 1997a,b; Fransen et al., 2002), the model suggests that entorhinal cells maintain a representation of the most recent experience as a signal for temporal context. Second, based on evidence that the entorhinal cortex participates in the formation of stimulus associations (Bunsey and Eichenbaum, 1993; Murray and Bussey, 1999), the model maintains that the entorhinal cortex retrieves sequential (forward-looking) locations associated with each location occupied by the rats as it traverses a route. According to the model, the information about temporal context and forward associations is sent to the hippocampus where they are integrated to support the selective retrieval of specific recent left- or right-turn episodes. Context-dependent activity and forward sequence retrieval within the entorhinal cortex should be reflected in the activity of entorhinal neurons as trial-type and location-specific activity, just as observed in hippocampal neurons. However, differences between entorhinal and hippocampal neurons may also be expected. To the extent that the main function of entorhinal processing is the representation of temporal context, it should be expected that entorhinal cells would strongly disambiguate leftand right-turn trials. At the same time, because the temporal context representation is based on persistent firing, less selective location-related firing should be expected from entorhinal neurons. In contrast, hippocampal representations that combine information about specific stimuli and behaviors that occur during an individual trial, may serve to sharpen the representation of each sequential event, reflected in more specific location-related firing within each trial (Eichenbaum et al., 1999).

The complementary functions of dcMEC and hippocampal CA1 neurons highlighted in this study suggest that these neuronal populations may each make distinct contributions to spatial navigation and memory. The findings suggest that the entorhinal cortex may be providing temporal context signals that disambiguate distinct spatial memories and that dcMEC neurons do so using a crude "sketch" of locations traversed in a route. The hippocampus sharpens the location representations, perhaps by combining spatial information with additional spatial and nonspatial information from other cortical inputs, to retrieve highly specific and therefore unambiguous sequence memories, and it contains information that links together related experiences.

\section{References}

Agster KL, Fortin NJ, Eichenbaum H (2002) The hippocampus and disambiguation of overlapping sequences. J Neurosci 22:5760-7568.

Ainge JA, van der Meer MA, Langston RF, Wood ER (2007) Exploring the role of context-dependent hippocampal activity in spatial alternation behaviour. Hippocampus, in press.

Bower MR, Euston DR, McNaughton BL (2005) Sequential-contextdependent hippocampal activity is not necessary to learn sequences with repeated elements. J Neurosci 25:1313-1323.

Bunsey M, Eichenbaum H (1993) Critical role of the parahippocampal region for paired-associate learning in rats. Behav Neurosci 107:740-747.

Burwell RD (2000) The parahippocampal region: corticocortical connectivity. Ann NY Acad Sci 911:25-42.

Dayan P, Abbott LF (2001) Theoretical neuroscience: computational and mathematical modeling of neural systems. Cambridge, MA: MIT.

Dolorfo CL, Amaral DG (1998) Entorhinal cortex of the rat: organization of intrinsic connections. J Comp Neurol 398:49-82.

Eichenbaum H, Dudchenko P, Wood ER, Shapiro M, Tanila H (1999) The hippocampus place cells, and memory: is it spatial memory or a memory space? Neuron 23:209-226.

Ferbinteanu J, Shapiro ML (2003) Prospective and retrospective memory coding in the hippocampus. Neuron 40:1227-1239.

Frank L, Brown EN, Wilson M (2000) Trajectory encoding in the hippocampus and entorhinal cortex. Neuron 27:169-178.

Frank LM, Brown EN, Wilson MA (2001) A comparison of the firing properties of putative excitatory and inhibitory neurons from CAl and the entorhinal cortex. J Neurophysiol 86:2029-2040.

Fransen E, Alonso AA, Hasselmo ME (2002) Simulations of the role of the muscarinic-activated calcium-sensitive nonspecific cation current INCM in entorhinal neuronal activity during delayed matching tasks. J Neurosci 22:1081-1097.

Freedman DJ, Riesenhuber M, Poggio T, Miller EK (2003) A comparison of primate prefrontal and inferior temporal cortices during visual categorization. J Neurosci 23:5235-5246.

Fyhn M, Molden S, Witter MP, Moser EI, Moser M (2004) Spatial representation in the entorhinal cortex. Science 305:1258-1264.

Fyhn M, Hafting T, Treves A, Moser MB, Moser EI (2007) Hippocampal remapping and grid realignment in entorhinal cortex. Nature 446:190194.

Gilbert PE, Kesner RP (2006) The role of the dorsal CA3 hippocampal subregion in spatial working memory and pattern separation. Behav Brain Res 169:142-149.

Hafting T, Fyhn M, Molden S, Moser M, Moser EI (2005) Microstructure of a spatial map in the entorhinal cortex. Nature 436:801-806.

Hargreaves EL, Rao G, Lee I, Knierim JJ (2005) Major dissociation between medial and lateral entorhinal input to dorsal hippocampus. Science 308:1792-1794.

Hasselmo ME, Eichenbaum H (2005) Hippocampal mechanisms for the context-dependent retrieval of episodes. Neural Netw 18:1172-1190.

Howard MW, Fotedar MS, Datey AS, Hasselmo ME (2005) The temporal context model in spatial navigation and relational learning: toward a common explanation of medial temporal lobe function across domains. Psychol Rev 112:75-116.

Kerr KM, Agster KL, Burwell R (2007) Functional neuroanatomy of the parahippocampal region: the lateral and medical entorhinal areas. Hippocampus, in press.

Klink R, Alonso A (1997a) Ionic mechanisms of muscarinic depolarization in entorhinal cortex layer II neurons. J Neurophysiol 77:1829-1843. 
Klink R, Alonso A (1997b) Muscarinic modulation of the oscillatory and repetitive firing properties of entorhinal cortex layer II neurons. J Neurophysiol 77:1813-1828.

Kumaran D, Maguire EA (2006) The dynamics of hippocampal activation during encoding of overlapping sequences. Neuron 49:617-629.

Lee I, Griffin AL, Zilli EA, Eichenbaum H, Hasselmo ME (2006) Gradual translocation of spatial correlates of neuronal firing in the hippocampus toward prospective reward locations. Neuron 51:639-650.

Lenck-Santini PP, Save E, Poucet B (2001) Place-cell firing does not depend on the direction of turn in a Y-maze alternation task. Eur J Neurosci 13:1055-1058.

Leutgeb JK, Leutgeb S, Moser, M-B and Moser EI (2007) Pattern separation in the dentate gyrus and CA3 of the hippocampus. Science 315:961.

Levy WB (1996) A sequence predicting CA3 is a flexible associator that learns and uses context to solve hippocampal-like tasks. Hippocampus 6:579-590.

Lisman JE (1999) Relating hippocampal circuitry to function: recall of memory sequences by reciprocal dentate-CA3 interactions. Neuron 22:233-242.

Markus EJ, Qin YL, Leonard B, Skaggs WE, McNaughton BL, Barnes CA (1995) Interactions between location and task affect the spatial and directional firing of hippocampal neurons. J Neurosci 15:7079-7094.

Mizumori SJ, Ward KE, Lavoie AM (1992) Medial septal modulation of entorhinal single unit activity in anesthetized and freely moving rats. Brain Res 570:188-197.

Muller RU, Kubie JL, Ranck Jr JB (1987) Spatial firing patterns of hippocampal complex-spike cells in a fixed environment. J Neurosci 7:1935-1950.
Murray EA, Bussey TJ (1999) Perceptual-mnemonic functions of the perirhinal cortex. Trends Cogn Sci 3:142-151.

Quirk GJ, Muller RU, Kubie JL, Ranck Jr., J. B (1992) The positional firing properties of medial entorhinal neurons: description and comparison with hippocampal place cells. J Neurosci 12:1945-1963.

Sargolini F, Fyhn M, Hafting T, McNaughton BL, Witter MP, Moser MB, Moser EI (2006) Conjunctive representation of position, direction, and velocity in entorhinal cortex. Science 312:758-762.

Skaggs WE, McNaughton BL, Gothard KM, Markus EJ (1993) An information-theoretic approach to deciphering the hippocampal code. In: Advances in neural information processing 5 (Hanson SJ, Cowan JD, Giles CL, eds), pp 1030-1037. San Mateo, CA: Morgan Kaufman.

Skaggs WE, McNaughton BL, Wilson MA, Barnes CA (1996) Theta phase precession in hippocampal neuronal populations and the compression of temporal sequences. Hippocampus 6:149-172.

Steffenach HA, Witter M, Moser MB, Moser EI (2005) Spatial memory in the rat requires the dorsolateral band of the entorhinal cortex. Neuron 45:301-313.

Tulving E (1983) Elements of episodic memory. New York: Oxford UP.

Vazdarjanova A, Guzowski JF (2004) Differences in hippocampal neuronal population responses to modifications of an environmental context: evidence for distinct, yet complementary, functions of CA3 and CA1 ensembles. J Neurosci 24:6489-6496.

Witter MP, Groenewegen HJ, Lopes de Silva FH, Lohman AH (1989) Functional organization of the extrinsic and intrinsic circuitry of the parahippocampal region. Prog Neurobiol 33:161-253.

Wood ER, Dudchenko PA, Robitsek RJ, Eichenbaum H (2000) Hippocampal neurons encode information about different types of memory episodes occurring in the same location. Neuron 27:623-633. 